ANALYSIS \& PDE Volume $7 \quad$ No.1 2014 (1) SŁAWOMIR DINEW AND SŁAWOMIR KOEODZIEJ

A PRIORI ESTIMATES FOR COMPLEX HESSIAN EQUATIONS 


\title{
A PRIORI ESTIMATES FOR COMPLEX HESSIAN EQUATIONS
}

\author{
SŁAwOmir Dinew And SŁAwomir KoŁodzieJ
}

\begin{abstract}
We prove some $L^{\infty}$ a priori estimates as well as existence and stability theorems for the weak solutions of the complex Hessian equations in domains of $\mathbb{C}^{n}$ and on compact Kähler manifolds. We also show optimal $L^{p}$ integrability for $m$-subharmonic functions with compact singularities, thus partially confirming a conjecture of Błocki. Finally we obtain a local regularity result for $W^{2, p}$ solutions of the real and complex Hessian equations under suitable regularity assumptions on the right-hand side. In the real case the method of this proof improves a result of Urbas.
\end{abstract}

\section{Introduction}

Hessian equations. Let $\lambda=\left(\lambda_{1}, \lambda_{2}, \ldots, \lambda_{n}\right)$ be the set of eigenvalues of a Hermitian $n \times n$ matrix $A$. By $S_{m}(A)$ denote the m-th elementary symmetric function of $\lambda$ :

$$
S_{m}(A)=\sum_{0<j_{1}<\cdots<j_{m} \leq n} \lambda_{j_{1}} \lambda_{j_{2}} \ldots \lambda_{j_{m}} .
$$

If $A$ is the complex Hessian of a real valued $C^{2}$ function $u$ defined in $\Omega \subset \mathbb{C}^{n}$ then we have a pointwise defined function

$$
\sigma_{m}\left(u_{z_{j} \bar{z}_{k}}\right)(z)=S_{m}\left(\left(u_{z_{j} \bar{z}_{k}}(z)\right)\right) .
$$

In terms of differential forms, with $d=\partial+\bar{\partial}, d^{c}=i(\bar{\partial}-\partial)$ and $\beta=d d^{c}\|z\|^{2}$ this function satisfies

$$
\left(d d^{c} u\right)^{m} \wedge \beta^{n-m}=\frac{m !(n-m) !}{n !} \sigma_{m}\left(u_{z_{j} \bar{z}_{k}}\right) \beta^{n} .
$$

We call a $C^{2}$ function $u: \Omega \rightarrow \mathbb{C}^{n} m$-subharmonic, or $m$-sh, if the forms

$$
\left(d d^{c} u\right)^{k} \wedge \beta^{n-k}
$$

are positive for $k=1, \ldots, m$ (in particular $u$ is subharmonic). If $u$ is subharmonic but not smooth, one can define $m$-sh function via inequalities for currents (see definitions in Section 1).

As shown by Błocki [2005] $m$-sh functions are the right class of admissible solutions to the complex Hessian equation

$$
\left(d d^{c} u\right)^{m} \wedge \beta^{n-m}=f \beta^{n}
$$

for a given nonnegative function $f$. Observe that for $m=1$ this is the Poisson equation and for $m=n$ the complex Monge-Ampère equation.

MSC2010: primary 32U15; secondary 32U05.

Keywords: Hessian equation, a priori estimate, pluripotential theory. 
Analogously, one can define $m$-subharmonic functions with respect to a Kähler form $\omega$ (abbreviatied $m-\omega$-sh) and the corresponding Hessian equation just replacing $\beta$ with $\omega$ in the preceding definitions. This definition can also be extended to subharmonic functions. Then one can consider such functions on Kähler manifolds.

Since on compact Kähler manifolds the sets of $m-\omega$-sh functions are trivial we define in this case $\omega$ - $m$-subharmonic $(\omega-m$-sh) functions requiring that

$$
\left(d d^{c} u+\omega\right)^{k} \wedge \omega^{n-k} \geq 0, \quad k=1, \ldots, m .
$$

and consider the Hessian equation on a compact Kähler manifold X, as in [Hou 2009; Hou et al. 2010; Dinew and Kołodziej 2012]:

$$
\left(d d^{c} u+\omega\right)^{m} \wedge \omega^{n-m}=f \omega^{n}, \quad \int_{X} f \omega^{n}=\int_{X} \omega^{n} .
$$

Solving the equation we look for $\omega$-m-sh solutions $u$. The normalization of $f$ is necessary because of Stokes' theorem and the Kähler condition $d \omega=0$.

Background. The real Hessian equation has been studied in many papers, for example [Caffarelli et al. 1985; Ivochkina et al. 2004; Krylov 1995; Trudinger 1995; Trudinger and Wang 1999; Labutin 2002; Chou and Wang 2001; Urbas 2001]. In particular the Dirichlet problem is solvable for smooth and strictly positive right-hand side under natural convexity assumptions on the boundary of the considered domain [Caffarelli et al. 1985]. This result is the starting point of study of degenerate Hessian equations [Ivochkina et al. 2004] and regularity of weak solutions [Urbas 2001]. A nonlinear potential theory has also been developed [Trudinger and Wang 1999; Labutin 2002]. We refer to [Wang 2009] for a survey of the real Hessian equation theory. It is interesting that the real and complex theories are very different, and attempts to apply "real" methods directly to the complex Hessian equation often fail. See [Błocki 2003; 2009] for a detailed study of those discrepancies.

The complex Hessian equation (0-1) in domains of $\mathbb{C}^{n}$ was first considered by S.-Y. Li [2004]. His main result says that if $\Omega$ is smoothly bounded and ( $m-1$ )-pseudoconvex (that means that $S_{j}, j=1, \ldots, m-1$, applied to the Levi form of $\partial \Omega$ are positive on the complex tangent to $\partial \Omega$ ) then, for smooth boundary data and for smooth, positive right-hand side there exists a unique smooth solution of the Dirichlet problem for the Hessian equation. The proof is in the spirit of the one in [Caffarelli et al. 1985].

Błocki [2005] considered also weak solutions of the equation, for possibly degenerate right-hand side, introducing some elements of potential theory for $m$-sh functions based on positivity of currents which are used in the definition. He proved that a $m$-sh function $u$ is maximal in this class if and only if

$$
\left(d d^{c} u\right)^{m} \wedge \beta^{n-m}=0 .
$$

Furthermore he described the maximal domain of definition of the Hessian operator.

As for the equation on compact Kähler manifolds (0-2), Hou [2009] has shown that the solutions, for smooth positive $f$, exist under the assumption that the metric has nonnegative holomorphic bisectional curvature. Similar results were independently obtained in [Kokarev 2010; Jbilou 2010]. Finally in [Dinew 
and Kołodziej 2012] the authors removed the curvature assumptions thus obtaining an analogue of the Calabi-Yau theorem for the complex Hessian equations.

New results. The $m$-subharmonic functions for $m<n$ are much more difficult to handle than the plurisubharmonic ones $(m=n)$. They lack a nice geometric description by the mean value property along planes, there is no invariance of the family under holomorphic mappings, and so forth. The cones of $\mathrm{m}-\omega$-sh functions are even worse - they are not invariant under translations. Despite that, the pluripotential theory methods developed in [Bedford and Taylor 1982; Kołodziej 1996; 1998; 2003] for the Monge-Ampère equation can be adapted to the Hessian equations. The crucial estimate between volume and capacity in Proposition 2.1 allowed us to prove a sharp integrability statement (conjectured in a stronger form in [Błocki 2005]): $m$-subharmonic functions, $m<n$, belong to $L^{q}$ for any $q<m n /(n-m)$, if their level sets are relatively compact in the domain where they are defined. For a plurisubharmonic function $u$ much stronger statement is true: $\exp (-a u)$ is locally integrable for some $a>0$. This accounts for the difference in statements of $L^{\infty}$ estimates for the Hessian equations and the Monge-Ampère equation. We show a priori $L^{\infty}$ bounds for the solutions of

$$
\left(d d^{c} u\right)^{m} \wedge \beta^{n-m}=f \omega^{n}
$$

(with continuous boundary data) and those of (0-2) with $f$ belonging to $L^{q}, q>n / m$. We also get strong stability theorems for those solutions. As a consequence one obtains that the families of solutions corresponding to data uniformly bounded in $L^{q}$ norms are equicontinuous.

The a priori estimates lead to the (continuous) solution of the Dirichlet problem in $(m-1)$-pseudoconvex domains for nonnegative right-hand side in the same $L^{q}$ spaces as above (Theorem 2.10). The corresponding existence result is also true on compact Kähler manifolds (Theorem 3.3). Those are the extensions of theorems in [Li 2004] and [Hou 2009]. Finally we prove the local regularity statement in Theorem 4.1 which in the case of the Monge-Ampère equation is due to Błocki and Dinew [2011]. It is worth noting that our methods applied to the real Hessian equations yield improvement of the regularity exponent obtained by Urbas [2001].

\section{Preliminaries}

We briefly recall the notions that we shall need later on. We start with a linear algebra toolkit.

Linear algebra preliminaries. Consider the set $M_{n}$ of all Hermitian symmetric $n \times n$ matrices. For a given matrix $M \in M_{n}$ let $\lambda(M)=\left(\lambda_{1}, \lambda_{2}, \ldots, \lambda_{n}\right)$ be its eigenvalues arranged in the decreasing order and let

$$
S_{k}(M)=S_{k}(\lambda(M))=\sum_{0<j_{1}<\cdots<j_{m} \leq n} \lambda_{j_{1}} \lambda_{j_{2}} \ldots \lambda_{j_{m}}
$$

be the $k$-th elementary symmetric polynomial applied to the vector $\lambda(M)$.

Then one can define the positive cones $\Gamma_{m}$ as

$$
\Gamma_{m}=\left\{\lambda \in \mathbb{R}^{n} \mid S_{1}(\lambda)>0, \ldots, S_{m}(\lambda)>0\right\} .
$$


The definition of $\Gamma_{m}$ is nonlinear if $m>1$; hence a priori it is unclear whether these sets are indeed convex cones. But the vectors in $\Gamma_{m}$, and hence the set of matrices with corresponding eigenvalues enjoy several convexity properties resembling the properties of positive definite matrices, and in particular the convexity of $\Gamma_{m}$.

Now let $V$ be a fixed positive definite Hermitian matrix and $\lambda_{i}(V)$ be the eigenvalues of a Hermitian matrix $M$ with respect to $V$. The we can analogously define the sets $\Gamma_{k}(V)$.

We list the properties of these cones that will be used later on:

(1) Maclaurin's inequality: If $\lambda \in \Gamma_{m}$ then $\left(S_{j} /\left(\begin{array}{l}n \\ j\end{array}\right)\right)^{1 / j} \geq\left(S_{i} /\left(\begin{array}{l}n \\ i\end{array}\right)\right)^{1 / i}$ for $1 \leq j \leq i \leq m$.

(2) Gårding's inequality [1959]: $\Gamma_{m}$ is a convex cone for any $m$ and the function $S_{m}^{1 / m}$ is concave when restricted to $\Gamma_{m}$.

(3) [Wang 2009]: Let $S_{k ; i}(\lambda):=S_{k}(\lambda)_{\lambda_{i}=0}=\frac{\partial S_{k+1}}{\partial \lambda_{i}}(\lambda)$. For any $\lambda, \mu \in \Gamma_{m}$,

$$
\sum_{i=1}^{n} \mu_{i} S_{m-1 ; i}(\lambda) \geq m S_{m}(\mu)^{1 / m} S_{m}(\lambda)^{(m-1) / m} .
$$

We refer to [Błocki 2005] or [Wang 2009] for further properties of these cones.

Potential theoretic aspects of $\boldsymbol{m}$-subharmonic functions. Let us fix a relatively compact domain $\Omega \in \mathbb{C}^{n}$. Let also $d=\partial+\bar{\partial}$ and $d^{c}:=i(\bar{\partial}-\partial)$ be the standard exterior differentiation operators. By $\beta:=d d^{c}\|z\|^{2}$ we denote the Euclidean Kähler form in $\mathbb{C}^{n}$.

Given a $\mathscr{b}^{2}(\Omega)$ function $u$ we call it $m-\beta$-subharmonic if for any $z \in \Omega$ the Hessian matrix $\left(\partial^{2} u / \partial z_{i} \partial \bar{z}_{j}\right)(z)$ has eigenvalues forming a vector in the closure of the cone $\Gamma_{m}$. Analogously if $\omega$ is any other Kähler form in $\Omega, u$ is $m$ - $\omega$-subharmonic if the Hessian matrix has eigenvalues at $z$ forming a vector in $\bar{\Gamma}_{m}(\omega(z))$ (the latter set will depend on $z$ in general).

Since the $\omega=\beta$ is the most natural case in the flat domains we shall call $m-\beta$-subharmonic functions just $m$-subharmonic or $m$-sh for short.

Observe that in the language of differential forms $u$ is $m$ - $\omega$-subharmonic if and only if the following inequalities hold:

$$
\left(d d^{c} u\right)^{k} \wedge \omega^{n-k} \geq 0, \quad k=1, \ldots, m .
$$

It was observed by Błocki [2005] that, following the ideas of Bedford and Taylor [1976; 1982], one can relax the smoothness requirement on $u$ and develop a nonlinear version of potential theory for Hessian operators.

The relevant definitions are as follows:

Definition 1.1. Let $u$ be a subharmonic function on a domain $\Omega \in \mathbb{C}^{n}$. Then $u$ is called $m$-subharmonic ( $m$-sh for short) if for any collection of $\mathfrak{C}^{2}$-smooth $m$-sh functions $v_{1}, \ldots, v_{m-1}$ the inequality

$$
d d^{c} u \wedge d d^{c} v_{1} \wedge \cdots \wedge d d^{c} v_{m-1} \wedge \beta^{n-m} \geq 0
$$

holds in the weak sense of currents. For a general Kähler form $\omega$ the notion of $m-\omega$-subharmonic function is defined by formally stronger condition: locally, in a neighborhood of any given point, there exists a 
decreasing to $u$ sequence of $\mathscr{C}^{2}$-smooth $m$ - $\omega$-sh functions $u_{j}$ such that for any set of $\mathscr{C}^{2}$-smooth $m$ - $\omega$-sh functions $v_{1}, \ldots, v_{m-1}$ the inequality

$$
d d^{c} u_{j} \wedge d d^{c} v_{1} \wedge \cdots \wedge d d^{c} v_{m-1} \wedge \omega^{n-m} \geq 0
$$

is satisfied. (For $\omega=\beta$ this condition is satisfied due to Proposition 1.3(4).)

The set of all $m$ - $\omega$-sh functions is denoted by $\mathscr{S H}_{m}(\omega, \Omega)$.

Remark 1.2. It is enough to test $m$-subharmonicity of $u$ against a collection of $m$-sh quadratic polynomials (see [Błocki 2005]).

Using the approximating sequence $u_{j}$ from the definition one can follow the Bedford and Taylor construction [1982] of the wedge products of currents given by locally bounded $m-\omega$-sh functions. They are defined inductively by

$$
u_{1} d d^{c} u_{2} \wedge \cdots \wedge d d^{c} u_{p} \wedge \omega^{n-m}:=d d^{c}\left(u_{1} \wedge \cdots \wedge d d^{c} u_{p} \wedge \omega^{n-m}\right) .
$$

It can be shown (see [Błocki 2005]) that analogously to the pluripotential setting these currents are continuous under monotone or uniform convergence of their potentials.

Here we list some basic facts about $m$-subharmonicity (assuming $\mathscr{C}^{2}$ smoothness).

Proposition 1.3. Let $\Omega \subset \mathbb{C}^{n}$ be a domain. Then:

(1) $\mathscr{S H}_{1}(\omega, \Omega) \subset \mathscr{S H}_{2}(\omega, \Omega) \subset \cdots \subset \mathscr{S} \mathscr{H}_{n}(\omega, \Omega)$.

(2) $\mathscr{S} \mathscr{H}_{m}(\omega, \Omega)$ is a convex cone.

(3) If $u \in \mathscr{Y H}_{m}(\omega, \Omega)$ and $\gamma: \mathbb{R} \rightarrow \mathbb{R}$ is a $\mathscr{C}^{2}$-smooth convex, increasing function then $\gamma \circ u \in \mathscr{Y} \mathcal{H}_{m}(\omega, \Omega)$.

(4) the standard regularizations $u * \rho_{\varepsilon}$ of a m-sh function is again m-sh.

Proof. The first claim is trivial. Second claim is proved in [Błocki 2005], with the use of Gårding's inequality [Gårding 1959]. Last two claims are more or less standard and their proofs are analogous to corresponding results for psh (plurisubharmonic) functions. Observe that the last property does fail for a general Kähler form $\omega$.

The following two theorems, known as comparison principles in pluripotential theory, follow essentially from the same arguments as in the case $m=n$ :

Theorem 1.4. Let $u, v$ be continuous $m$ - $\omega$-sh functions in a domain $\Omega \in \mathbb{C}^{n}$. Suppose that

$$
\liminf _{z \rightarrow \partial \Omega}(u-v)(z) \geq 0 .
$$

Then

$$
\int_{\{u<v\}}\left(d d^{c} v\right)^{m} \wedge \omega^{n-m} \leq \int_{\{u<v\}}\left(d d^{c} u\right)^{m} \wedge \omega^{n-m} .
$$

Theorem 1.5. Let $u, v$ be continuous $m-\omega$-sh functions in a domain $\Omega \in \mathbb{C}^{n}$. Suppose that

$$
\liminf _{z \rightarrow \partial \Omega}(u-v)(z) \geq 0 \quad \text { and } \quad\left(d d^{c} v\right)^{m} \wedge \omega^{n-m} \geq\left(d d^{c} u\right)^{m} \wedge \omega^{n-m} .
$$

Then $v \leq u$ in $\Omega$. 
The last result yields, in particular, uniqueness of bounded weak solutions of the Dirichlet problem. As for the existence we have the following fundamental theorem:

Theorem 1.6 [Li 2004]. Let $\Omega$ be a smoothly bounded relatively compact domain in $\mathbb{C}^{n}$. Suppose that $\partial \Omega$ is $(m-1)$-pseudoconvex (that means that Levi form at any point $p \in \partial \Omega$ has its eigenvalues in the cone $\left.\Gamma_{m-1}\right)$. Let $\varphi$ be a smooth function on $\partial \Omega$ and $f$ a strictly positive and smooth function in $\Omega$. Then the Dirichlet problem

has a smooth solution $u$.

$$
\left\{\begin{array}{l}
u \in \mathscr{P} \mathcal{H}_{m}(\Omega, \beta) \cap \mathscr{C}(\bar{\Omega}), \\
\left(d d^{c} u\right)^{m} \wedge \beta^{n-m}=f, \\
\left.u\right|_{\partial \Omega}=\varphi
\end{array}\right.
$$

The convexity properties of the cones $\Gamma_{m}$ yield the following mixed Hessian inequalities:

Proposition 1.7. Let $u_{1}, \ldots, u_{m}$ be $m-s h \mathcal{C}^{2}$ functions in a domain $\Omega \in \mathbb{C}^{n}$. Suppose $\left(d d^{c} u_{j}\right)^{m} \wedge \beta^{n-m}=f_{j}$ for some continuous nonnegative functions $f_{j}$. Then

$$
d d^{c} u_{1} \wedge \cdots \wedge d d^{c} u_{m} \wedge \beta^{n-m} \geq\left(f_{1} \cdots f_{m}\right)^{1 / m} \beta^{n} .
$$

Proof. Pointwise this reduces to the Gårding inequality; see also (1-2) for the case $u_{2}=u_{3}=\cdots=u_{m}$.

Later on in Theorem 2.12 we shall see that the smoothness assumptions here can be considerably relaxed.

Kähler setting. Given a compact Kähler manifold $(X, \omega)$ we can define the cones $\mathscr{S} \mathscr{H}_{m}(X, \omega)$ consisting of those functions $u$ for which, in a local chart $\Omega$ where $\omega$ has a potential $\rho$, the function $u+\rho$ belongs to $\mathscr{Y} \mathcal{H}_{m}(\Omega, \omega)$. The definition is independent of the choice of the chart and the potential. This essentially allows us to carry over all local results to this setting. We refer to [Kołodziej 2005] for the plurisubharmonic $(m=n)$ case.

The Dirichlet problem for smooth nondegenerate data was recently solved:

Proposition 1.8 [Dinew and Kołodziej 2012]. Let $(X, \omega)$ be a compact Kähler manifold and $1<m<n$ be an integer number. Given a strictly positive smooth function $f$ satisfying the condition $\int_{X} f \omega^{n}=\int_{X} \omega^{n}$ there is an unique function $u \in \mathscr{Y H}_{m}(X, \omega) \cap \mathscr{C}^{\infty}(X)$ solving the Dirichlet problem

$$
\left(d d^{c} u+\omega\right)^{m} \wedge \omega^{n-m}=f \omega^{n}, \quad \sup _{X} u=0 .
$$

The comparison principle on compact manifolds reads as follows:

Proposition 1.9. Let $(X, \omega)$ be a compact Kähler manifold and $u, v$ continuous functions in $\mathscr{S H}_{m}(X, \omega)$. Then

$$
\int_{\{u<v\}}\left(\omega+d d^{c} v\right)^{m} \wedge \omega^{n-m} \leq \int_{\{u<v\}}\left(\omega+d d^{c} u\right)^{m} \wedge \omega^{n-m} .
$$

Proof. One can repeat the proof for psh functions from [Kołodziej 2003] or [Kołodziej 2005].

Observe that the cones $\Gamma_{k}(\omega)$ are not fixed but according to an observation of Hou [2009] these are invariant under the parallel transport defined by the Levi-Civita connection associated to $\omega$. 


\section{2. $L^{\infty}$ estimates and existence of weak solutions in domains}

In this section we state the results for $0<m<n$. Let us denote by $B(a, r)$ the ball in $\mathbb{C}^{n}$ with center $a$ and radius $r$. Let also $\omega$ be a Kähler form defined in a neighborhood of the closure of a set $\Omega$ considered below and $V=\omega^{n}$ be the volume form associated to $\omega$.

Let $\mathscr{S} \mathcal{H}_{m}(\omega, \bar{\Omega})$ denote the class of $m$ - $\omega$-sh functions which are continuous in $\bar{\Omega}$.

Proposition 2.1. For $p<n /(n-m)$ and an open set $\Omega \subset B(0,1)=B$ there exists $C(p)$ such that for any $K \Subset \Omega$,

$$
V(K) \leq C(p) \operatorname{cap}_{m}^{p}(K, \Omega)
$$

where

$$
\operatorname{cap}_{m}(K, \Omega)=\sup \left\{\int_{K}\left(d d^{c} u\right)^{m} \wedge \omega^{n-m}, u \in \mathscr{Y} \mathscr{H}_{m}(\omega, \bar{\Omega}), 0 \leq u \leq 1\right\} .
$$

Proof. If $V(K)=0$ then the inequality trivially holds. Assume from now on that $V(K)>0$. Fix any $\epsilon \in(0,1 / 2)$ and set $f=[V(K)]^{2 \epsilon-1} \chi_{K}$, where $\chi_{K}$ denotes the characteristic function of the set $K$. Solve the complex Monge-Ampère equation in $B$ to find $v \in P S H_{\omega}(B) \cap C(\bar{\Omega})$ with $v=0$ on $\partial B$ and

$$
\left(d d^{c} v\right)^{n}=f \omega^{n} .
$$

By the inequality between mixed Monge-Ampère measures (see [Kołodziej 2005; Dinew 2009])

$$
\left(d d^{c} v\right)^{m} \wedge \omega^{n-m} \geq[V(K)]^{(2 \epsilon-1) m / n} \chi_{K} \omega^{n} .
$$

For $q=1+\epsilon$

$$
\int_{B} f^{q} d V=[V(K)]^{(2 \epsilon-1)(1+\epsilon)+1}=[V(K)]^{\epsilon+2 \epsilon^{2}} \leq V(B) .
$$

So, by [Kołodziej 1996], there exists $c>0$, independent of $K$ (though dependent on $\epsilon$ ), such that $\|v\| \leq 1 / c$. Take $u=c v$. Then, using (2-1)

$$
\operatorname{cap}_{m}(K, \Omega) \geq \int_{K}\left(d d^{c} u\right)^{m} \wedge \omega^{n-m} \geq c^{m}[V(K)]^{(2 \epsilon-1)(m / n)+1} .
$$

Therefore

$$
V(K) \leq C \operatorname{cap}_{m}^{n /(n-m+2 m \epsilon)}(K, \Omega),
$$

which proves the claim.

Proposition 2.2. Let $\Omega$ and $p$ be as above and consider $u \in \mathscr{S H}_{m}(\omega, \bar{\Omega})$ with $u=0$ on $\partial \Omega$ and

$$
\int_{\Omega}\left(d d^{c} u\right)^{m} \wedge \omega^{n-m} \leq 1 .
$$

Then for $U(s)=\{u<-s\}$ we have

$$
\operatorname{cap}_{m}(U(s), \Omega) \leq s^{-m} \quad \text { and } \quad V_{2 n}(U(s)) \leq C(p) s^{-p m} .
$$

In particular $u \in L^{q}(\Omega)$ for any $q<m n /(n-m)$, and this remains true whenever $u$ is bounded in some neighborhood of the boundary of $\Omega$. 
Proof. Fix $\epsilon>0, t>1$ and $K \subset U(s)$ and find $v \in \mathscr{S} \mathscr{H}_{m}(\omega, \bar{\Omega})$ with $-1 \leq v \leq 0$ and

$$
\int_{K}\left(d d^{c} v\right)^{m} \wedge \omega^{n-m} \geq \operatorname{cap}_{m}(K, \Omega)-\epsilon .
$$

Then, using the comparison principle [Bedford and Taylor 1976; Błocki 2005], we obtain

$\operatorname{cap}_{m}(K, \Omega)-\epsilon \leq \int_{K}\left(d d^{c} v\right)^{m} \wedge \omega^{n-m} \leq \int_{\left\{-\frac{t}{s} u<v\right\}}\left(d d^{c} v\right)^{m} \wedge \omega^{n-m} \leq\left(\frac{t}{s}\right)^{m} \int_{\Omega}\left(d d^{c} u\right)^{m} \wedge \omega^{n-m} \leq\left(\frac{t}{s}\right)^{m}$.

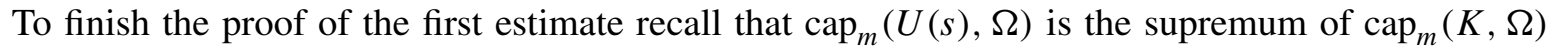
over compact $K \subset U(s)$ and let $\epsilon \rightarrow 0$ and $t \rightarrow 1$. Then the estimate of the volume follows from Proposition 2.1.

Remark 2.3. The bound for $q$ above is optimal as the function

$$
G(z)=-|z|^{2-2 n / m}
$$

is $m$-sh and belongs to $L_{l o c}^{q}$ if and only if $q<m n /(n-m)$.

Błocki [2005] conjectured that any $m$-sh function belongs to $L_{\text {loc }}^{q}(\Omega)$ for any $q<m n /(n-m)$. He proved this for $q<n /(n-m)$. The above proposition confirms partially the conjecture - under the extra assumption of boundedness near the boundary. Still the question about the local integrability remains open.

We now proceed to proving the $L^{\infty}$ a priori estimates for the Hessian equation with the right-hand side controlled in terms of the capacity.

Lemma 2.4. For $p \in(1, n /(n-m))$ and an open set $\Omega \subset B$ consider $u, v \in \mathscr{S}_{m}(\omega, \bar{\Omega})$ satisfying

$$
\int_{K}\left(d d^{c} u\right)^{m} \wedge \omega^{n-m} \leq A \operatorname{cap}_{m}^{p}(K, \Omega)
$$

for some $A>0$ and any compact $K \subset \Omega$. If the sets $U(s)=\{u-s<v\}$ are nonempty and relatively compact in $\Omega$ for $s \in\left(s_{0}, s_{0}+t_{0}\right)$ then there exists a constant $C(p, A)$ such that

$$
t_{0} \leq C(p, A) \operatorname{cap}_{m}^{p / n}\left(U\left(s_{0}+t_{0}\right), \Omega\right) .
$$

Proof. Using the notation

$$
a(s)=\operatorname{cap}_{m}(U(s), \Omega), \quad b(s)=\int_{U(s)}\left(d d^{c} u\right)^{m} \wedge \omega^{n-m}
$$

we claim that

$$
t^{m} a(s) \leq b(s+t), \quad t \in\left(0, s_{0}+t_{0}-s\right) .
$$

Indeed, for fixed compact $K \subset U(s)$, take $w_{1} \in \mathscr{S H}_{m}(\omega, \bar{\Omega}),-1 \leq w_{1} \leq 0$, such that

$$
\int_{K}\left(d d^{c} w_{1}\right)^{m} \wedge \omega^{n-m} \geq \operatorname{cap}_{m}(K, \Omega)-\epsilon .
$$

Then for $w_{2}=(u-s-t) / t$ one readily verifies that $K \subset V \subset U(s+t)$, where $V=\left\{w_{2}<w_{1}+v / t\right\}$. 
So, by the comparison principle,

$$
\begin{aligned}
\operatorname{cap}_{m}(K, \Omega)-\epsilon & \leq \int_{K}\left(d d^{c}\left(w_{1}+\frac{1}{t} v\right)\right)^{m} \wedge \omega^{n-m} \leq \int_{V}\left(d d^{c}\left(w_{1}+\frac{1}{t} v\right)\right)^{m} \wedge \omega^{n-m} \\
& \leq \int_{V}\left(d d^{c} w_{2}\right)^{m} \wedge \omega^{n-m} \leq t^{-m} b(s+t)
\end{aligned}
$$

Having (2-2) one proceeds as in the proof of Lemma 4.3 in [Kołodziej 2002] (with $\left.h(x)=x^{m(p-1)}\right)$ to reach the conclusion.

Coupling this with the volume estimate in Proposition 2.1 we obtain a priori estimates for the solutions of Hessian equations with the right-hand side in some $L^{q}$ spaces.

Theorem 2.5. Take $q>n / m$. Then the conjugate $q^{\prime}$ of $q$ satisfies $q^{\prime}<n /(n-m)$. Fix $p^{\prime} \in\left(q^{\prime}, n /(n-m)\right)$ and $p=p^{\prime} / q^{\prime}>1$. Consider $u, v \in \mathscr{S}_{\mathcal{H}_{m}}(\omega, \bar{\Omega})$ such that $u \geq v$ on $\partial \Omega,\{u<v\} \neq \varnothing$ and

$$
\left(d d^{c} u\right)^{m} \wedge \omega^{n-m}=f \omega^{n},
$$

for some $f \in L^{q}(\Omega, d V)$. Then

$$
\sup (v-u) \leq c\left(p^{\prime}, q,\|f\|_{L^{q}(\Omega)}\right)\left\|(v-u)_{+}\right\|_{L^{q^{\prime}}(\Omega)}^{p /(n+p(m+1))}, \quad(v-u)_{+}:=\max (v-u, 0) .
$$

Proof. By the Hölder inequality and Proposition 2.1, for a compact set $K \subset \Omega$ we have

$$
\int_{K} f \omega^{n} \leq\|f\|_{q} V(K)^{1 / q^{\prime}} \leq C(p)\|f\|_{L^{q}(\Omega)} \operatorname{cap}_{m}^{p}(K, \Omega) .
$$

Therefore, by Lemma 2.4, we get for $t=\frac{1}{2} \sup (v-u)$ and $E(t)=\{u+t<v\}$,

$$
t \leq c\left(p^{\prime}, q,\|f\|_{L^{q}(\Omega)}\right) \operatorname{cap}_{m}^{p / n}(E(t), \Omega) .
$$

To shorten notation set $a(t)=\operatorname{cap}_{m}(E(2 t), \Omega)$. Take $w \in \mathscr{S}_{m}(\omega, \bar{\Omega}),-1 \leq w \leq 0$ such that

$$
\int_{E(2 t)}\left(d d^{c} w\right)^{m} \wedge \omega^{n-m} \geq \frac{1}{2} a(t) .
$$

Observe that for $V=\{u<t w+v-t\}$ we have $E(2 t) \subset V \subset E(t)$. Applying the comparison principle we thus get

$$
\frac{1}{2} a(t) t^{m} \leq \int_{E(2 t)}\left[d d^{c}(t w+v)\right]^{m} \omega^{n-m} \leq \int_{V}\left(d d^{c} u\right)^{m} \wedge \omega^{n-m} \leq \int_{E(t)} f d V .
$$

Hence from the Hölder inequality one infers

$$
a(t) t^{m+1} \leq 2 \int_{\Omega}(v-u)_{+} f d V \leq\|f\|_{L^{q}(\Omega)}\left\|(v-u)_{+}\right\|_{q^{\prime}} .
$$

Inserting this estimate into (2-3) we arrive at

$$
t \leq c_{1}\left(p^{\prime}, q,\|f\|_{L^{q}(\Omega)}\right)\left[\|f\|_{q}\left\|(v-u)_{+}\right\|_{L^{q^{\prime}}(\Omega)} t^{-m-1}\right]^{p / n},
$$

and consequently

$$
t \leq c_{2}\left(p^{\prime}, q,\|f\|_{L^{q}(\Omega)}\right)\left\|(v-u)_{+}\right\|_{L^{q^{\prime}}(\Omega)}^{p /(n+p(m+1))} .
$$


Corollary 2.6. The last theorem gives a priori $L^{\infty}$ estimate for the solutions of the Hessian equation (0-3) with the right-hand side in $L^{q}$ and a fixed boundary condition.

Indeed, we apply the theorem for the solution $u$ of

$$
\left(d d^{c} u\right)^{m} \wedge \omega^{n-m}=f \omega^{n},
$$

with given continuous boundary data $\varphi$ and for $v$, which is the maximal function in $\mathscr{S}_{\mathcal{H}_{m}}(\omega, \bar{\Omega})$ matching the boundary condition (it exists by [Błocki 2005]). Then $u$ is bounded by a constant depending on $\Omega,\|\varphi\|=\|v\|$, and $\|f\|_{q}$ since $\left\|(v-u)_{+}\right\|_{L^{q^{\prime}}(\Omega)}$ is bounded (Proposition 2.2).

Corollary 2.7. The solutions of the Hessian equation with the right-hand sides uniformly bounded in $L^{q}$ $q>n / m$ and given continuous boundary data form an equicontinuous family.

Proof. As in [Kołodziej 2005, p. 35], which deals with the Monge-Ampère case.

Below we state yet another stability theorem which we shall need later. Given the estimates we have already proven its proof follows the arguments from [Kołodziej 1996].

Theorem 2.8. Let $q>n / m$. Consider $u, v \in \mathscr{S}^{\mathcal{H}_{m}}(\omega, \bar{\Omega})$ such that $\{u<v\} \neq \varnothing$ and

$$
\left(d d^{c} u\right)^{m} \wedge \omega^{n-m}=f \omega^{n}, \quad\left(d d^{c} v\right)^{m} \wedge \omega^{n-m}=g \omega^{n}
$$

for some $f, g \in L^{q}(\Omega, d V)$. Then

$$
\sup _{\Omega}(v-u) \leq \sup _{\partial \Omega}(v-u)+c(q, m, n, \operatorname{diam}(\Omega))\|f-g\|_{L^{q}(\Omega)}^{1 / m} .
$$

Remark 2.9. The analogous stability theorem for the real $m$-Hessian equation $(m<n / 2)$ can be found in [Wang 2009, Theorem 5.5] (see also [Chou and Wang 2001]). There the optimal exponent $q$ is equal to $n / 2 m$.

Next we obtain a theorem on the existence of weak, continuous solutions when $\omega=\beta$ and the right-hand side is in $L^{q}, q>n / m$.

Theorem 2.10. Let $\Omega$ be smoothly bounded (m-1)-pseudoconvex domain (as in Theorem 1.6). Then for $q>n / m, f \in L^{q}(\Omega, d V)$ and continuous $\varphi$ on $\partial \Omega$ there exists $u \in \mathscr{S H}_{m}(\omega, \bar{\Omega})$ satisfying

$$
\left(d d^{c} u\right)^{m} \wedge \beta^{n-m}=f \beta^{n}
$$

and $u=\varphi$ on $\partial \Omega$.

Proof. For smooth, positive $f$ this is the result of Li [2004] (Theorem 1.6). With our assumptions we approximate $f$ in $L^{q}(\Omega, d V)$ by smooth positive $f_{j}$ and approximate uniformly $\varphi$ by smooth $\varphi_{j}$. The solutions $u_{j}$ corresponding to $f_{j}, \varphi_{j}$ are equicontinuous and uniformly bounded (Corollaries 2.6, 2.7). Thus we can pick up a subsequence converging uniformly to some $u \in \mathscr{S}_{\mathcal{H}_{m}}(\omega, \bar{\Omega})$. By the convergence theorem $u$ solves the equation.

Remark 2.11. For $\omega=\beta$, the plurisubharmonic function $u(z)=\log \|z\|$ has a $m$-Hessian density in $L^{p}$ for any $p<n / m$ which shows that the exponent $n / m$ is optimal. 
Equipped with the existence and stability of weak solutions we can also prove the weak Gårding inequality announced in Section 1:

Theorem 2.12. Let $u_{1}, \ldots, u_{m}$ be locally bounded $m$-sh functions in some domain $\Omega \in \mathbb{C}^{n}$. Suppose $\left(d d^{c} u_{j}\right)^{m} \wedge \beta^{n-m}=f_{j} \beta^{n}$ for some nonnegative functions $f_{j} \in L^{q}(\Omega), q>n / m$. Then

$$
d d^{c} u_{1} \wedge \wedge \cdots \wedge d d^{c} u_{m} \wedge \beta^{n-m} \geq\left(f_{1} \cdots f_{m}\right)^{1 / m} \beta^{n} .
$$

Proof. We can essentially follow the lines of the proof of the analogous result for psh functions from [Kołodziej 2003] (see also [Kołodziej 2005]). First observe that the inequality is purely local hence it suffices to prove it under the additional assumptions that $\Omega$ is a ball and all the functions $u_{i}$ are defined in a slightly bigger ball. Hence one can use convolutions with smoothing kernel to produce a decreasing to $u_{i}$ sequence of $m$-sh functions $\left\{u_{i, j}\right\}_{j=1}^{\infty}$ (compare Proposition 1.3). Then given any collection of smooth positive functions $f_{i, k} \in L^{q}(\Omega), q>n / m$, by [Li 2004] we can solve the Dirichlet problems

$$
\left\{\begin{array}{l}
v_{i, j, k} \in \mathscr{P H}_{m}(\Omega) \cap \mathscr{C}^{\infty}(\Omega), \\
\left(d d^{c} v_{i, j, k}\right)^{m} \wedge \beta^{n-m}=f_{i, k} \beta^{n}, \\
\left.v_{i, j, k}\right|_{\partial \Omega}=u_{i, j} .
\end{array}\right.
$$

For those smooth functions we can apply pointwise the Gårding inequality to conclude that

$$
d d^{c} v_{1, j, k} \wedge \cdots \wedge d d^{c} v_{m, j, k} \wedge \beta^{n-m} \geq\left(f_{1, k} \cdots f_{m, k}\right)^{1 / m} \beta^{n}
$$

for any $j, k \geq 1$. Then given any nonnegative $f_{i} \in L^{q}(\Omega), q>n / m$, we can find an approximating sequence of smooth positive $\left\{f_{i, k}\right\}_{k=1}^{\infty}$ which converge in $L^{q}$ to $f_{i}$. By the stability theorem the corresponding solutions $v_{i, j, k}$ (recall they the same boundary values $u_{i, j}$ ) converge uniformly as $k \rightarrow \infty$ to the $m$-sh functions $v_{i, j}$ (solving the limiting weak equation), and hence the inequality follows from the continuity of Hessian currents under uniform convergence of their potentials. Now if we let $j \rightarrow \infty$ the boundary values decrease towards $u_{i}$ and hence so do the functions $v_{i, j}$ by the comparison principle. The convergence is not uniform but monotonicity is still sufficient to guarantee the continuity and hence in the limit we obtain the claimed inequality.

Remark 2.13. The weak Gårding inequality can be further generalized similarly to the $m=n$ case as in [Dinew 2009].

\section{3. $L^{\infty}$ estimates and existence of weak solutions on compact Kähler manifolds}

The a priori estimates from the previous section can be carried over to the case of compact Kähler manifolds as it was done in [Kołodziej 2003] or [2005] for the Monge-Ampère equation. Let us consider a compact n-dimensional Kähler manifold $X$ equipped with the fundamental form $\omega$ and recall that a continuous function $u$ is $\omega$-m-subharmonic ( $\omega$-m-sh) on $X$ if

$$
\left(\omega+d d^{c} u\right)^{k} \wedge \omega^{n-k} \geq 0, \quad k=1,2, \ldots, m .
$$


The set of such functions is denoted by $\mathscr{S H}_{m}(X, \omega)$. We study the complex $m$-Hessian equation

$$
\left(\omega+d d^{c} u\right)^{m} \wedge \omega^{n-m}=f \omega^{n}
$$

with given nonnegative function $f \in L^{1}(M)$, which is normalized by the condition

$$
\int_{X} f \omega^{n}=\int_{X} \omega^{n}
$$

The solution is required to be $\omega-m$-sh. By Proposition 1.8 the solutions of the equation exist, at least for smooth positive $f$. Our a priori estimates will also give the existence of weak solutions for $f \geq 0$ in $L^{q}$, $q>n / m$.

We define for a compact set $K \subset X$ its capacity

$$
\operatorname{cap}_{m}(K)=\sup \left\{\int_{K}\left(\omega+d d^{c} u\right)^{m} \wedge \omega^{n-m}: u \in \mathscr{P} \mathscr{H}_{m}(X, \omega), 0 \leq u \leq 1\right\} .
$$

To use the local results we need also a capacity defined as follows. Let us consider two finite coverings by strictly pseudoconvex sets $\left\{B_{s}\right\},\left\{B_{s}^{\prime}\right\}, s=1,2, \ldots, N$, of $X$ such that $\bar{B}_{s}^{\prime} \subset B_{s}$ and in each $B_{s}$ there exists $v_{s} \in P S H\left(B_{s}\right)$ with $d d^{c} v_{s}=\omega$ and $v_{s}=0$ on $\partial B_{s}$. Given a compact set $K \subset X$ define $K_{s}=K \cap \bar{B}_{s}^{\prime}$. Set

$$
\operatorname{cap}_{m}^{\prime}(K)=\sum_{s} \operatorname{cap}_{m}\left(K_{s}, B_{s}\right)
$$

where $\operatorname{cap}_{m}(K, B)$ denotes the relative capacity from the previous section. As in [Kołodziej 2003] one can show that $\operatorname{cap}_{m}(K)$ is comparable with $\operatorname{cap}_{m}^{\prime}(K)$ : There exists $C>0$ such that

$$
\frac{1}{C} \operatorname{cap}_{m}(K) \leq \operatorname{cap}_{m}^{\prime}(K) \leq C \operatorname{cap}_{m}(K) .
$$

Hence, by Proposition 2.1 we have

$$
V(K) \leq C(p, X) \operatorname{cap}_{m}^{p}(K),
$$

for $p<n /(n-m)$ and $V$ the volume measured by $\omega^{n}$.

With this estimate at our disposal we can obtain the same a priori estimates as in domains in $\mathbb{C}^{n}$. The proofs are almost identical. In the compact setting one has to make sure that instead of just a sum of $m$-sh functions one considers a convex combination of $\omega$ - $m$-sh functions (see [Kołodziej 2005]). In particular the following theorems hold.

Theorem 3.1. Consider $q>n / m$, its conjugate $q^{\prime}$ and $p^{\prime} \in\left(q^{\prime}, n /(n-m)\right)$. Write $p=p^{\prime} / q^{\prime}>1$. Consider $u, v \in \mathscr{S}^{\mathcal{H}_{m}}(X, \omega)$ such that $\{u<v\} \neq \varnothing$ and

$$
\left(\omega+d d^{c} u\right)^{m} \wedge \omega^{n-m}=f \omega^{n},
$$

for some $f \in L^{q}(d V)$. Then

$$
\sup (v-u) \leq c\left(p^{\prime}, q,\|f\|_{L^{q}(X)}\right)\left\|(v-u)_{+}\right\|_{q^{\prime}}^{\frac{p}{n+p(m+1)}}, \quad \text { where }(v-u)_{+}:=\max (v-u, 0) .
$$


Corollary 3.2. The family of solutions of the Hessian equation (3-1) with right-hand sides uniformly bounded in $L^{q}, q>n / m$, is equicontinuous.

Applying Proposition 1.8 and the statements above one immediately gets this existence theorem:

Theorem 3.3. Let $X$ be a compact Kähler manifold. For $q>n / m$ and $f \in L^{q}(d V)$ there exists a unique function $u \in \mathscr{S}^{\mathcal{H}_{m}}(X, \omega)$ satisfying

$$
\left(\omega+d d^{c} u\right)^{m} \wedge \omega^{n-m}=f \omega^{n} \quad \text { and } \quad \max u=0 .
$$

\section{Local regularity}

In this section we prove a counterpart of the main result in [Błocki and Dinew 2011], where the case of the Monge-Ampère equation was studied. We shall treat only the $\omega=\beta$ case and use PDE notation (with $\sigma_{m}$ defined in the Introduction).

Theorem 4.1. Assume that $n \geq 2$ and $p>n(m-1)$. Let $u \in W^{2, p}(\Omega)$, where $\Omega$ is a domain in $\mathbb{C}^{n}$, be a $m$-subharmonic solution of

$$
\sigma_{m}\left(u_{z_{j} \bar{z}_{k}}\right)=\psi>0 .
$$

Assume that $\psi \in C^{1,1}(\Omega)$. Then for $\Omega^{\prime} \Subset \Omega$

$$
\sup _{\Omega^{\prime}} \Delta u \leq C,
$$

where $C$ is a constant depending only on $n, m, p$, $\operatorname{dist}\left(\Omega^{\prime}, \partial \Omega\right), \inf _{\Omega} \psi, \sup _{\Omega} \psi,\|\psi\|_{C^{1,1}(\Omega)}$ and $\|\Delta u\|_{L^{p}(\Omega)}$.

Proof. By $C_{1}, C_{2}, \ldots$ we will denote possibly different constants depending only on the required quantities. Without loss of generality we may assume that $\Omega=B$ is the unit ball in $\mathbb{C}^{n}$ and that $u$ is defined in some neighborhood of $\bar{B}$. We will use the notation $u_{j}=u_{z_{j}}, u_{\bar{j}}=u_{\bar{z}_{j}}$ with the notable exception of $u_{(\epsilon)}$ which is defined below.

Following [Bedford and Taylor 1976], we define the Laplacian approximating operator

where

$$
T=T_{\epsilon}(u)=\frac{n+1}{\epsilon^{2}}\left(u_{(\epsilon)}-u\right),
$$

$$
u_{(\epsilon)}(z)=\frac{1}{V(B(z, \epsilon))} \int_{B(z, \epsilon)} u d V
$$

Since $T_{\epsilon} u \rightarrow \Delta u$ weakly as $\epsilon \rightarrow 0$, it is enough to show a uniform upper bound for $T$ independent of $\epsilon$. Observe that since $u$ is subharmonic we have $T_{\epsilon}(u) \geq 0$.

Before we continue let us state two lemmas. The first one is classical.

Lemma 4.2. Let $u \in W^{2, p}(\Omega)$ ( $\Omega$ is a domain in $\mathbb{C}^{n}$ ) be a subharmonic function. Given any $\Omega^{\prime} \Subset \Omega$ the operator $T_{\epsilon}(z)$ is well defined on $\Omega^{\prime}$ for any sufficiently small $\epsilon>0$. Furthermore,

$$
\left\|T_{\epsilon}\right\|_{L^{p}\left(\Omega^{\prime}\right)} \rightarrow\|\Delta u\|_{L^{p}\left(\Omega^{\prime}\right)} ;
$$

in particular, $\left\|T_{\epsilon}\right\|_{L^{p}\left(\Omega^{\prime}\right)}$ is uniformly bounded for all $0<\epsilon<\epsilon_{0}$. 
Lemma 4.3. The function $T_{\epsilon}(u)(z)$ for any $\epsilon>0$ satisfies the subharmonicity condition

$$
\frac{\partial \sigma_{m}\left(u_{j \bar{k}}\right)}{\partial u_{i \bar{j}}} T_{\epsilon, i \bar{j}} \geq-C_{1},
$$

where $\left.\left(\partial \sigma_{m}\left(u_{j}\right)\right)\right) / \partial u_{i \bar{j}}$ is the $(i, j)$-th $(m-1)$-cominor of the matrix $u_{i \bar{j}}(z)$ and $C_{1}$ is a constant dependent only on $n, m, \inf _{\Omega} \psi, \sup _{\Omega} \psi$, and $\|\psi\|_{C^{1,1}(\Omega)}$.

Proof. Observe that $u_{(\epsilon)}$ is a convex combination of $m$-subharmonic functions, hence it is $m$-subharmonic. Therefore one has the inequality

$$
\left(d d^{c} u_{(\epsilon)}\right)^{m} \wedge \omega^{n-m} \geq 0
$$

In fact following the lines of the same argument in [Bedford and Taylor 1976] (where it was applied to the Monge-Ampère operator) one can prove the stronger inequality

$$
\left(d d^{c} u_{(\epsilon)}\right)^{m} \wedge \omega^{n-m} \geq\left(\left(\psi^{1 / m}\right)_{(\epsilon)}\right)^{m}
$$

Indeed, for smooth $u$ this is just a consequence of the concavity of $\sigma_{m}^{1 / m}$. For nonsmooth solutions one can repeat the Goffman-Serrin formalism, again following Bedford and Taylor.

Thus using the weak Gårding inequality (Theorem 2.12) one has

$$
\left(d d^{c} u\right)^{m-1} \wedge d d^{c} u_{(\epsilon)} \wedge \omega^{n-m} \geq \psi^{(m-1) / m}\left(\psi^{1 / m}\right)_{(\epsilon)} d V
$$

Next, identifying $(n, n)$ forms and their densities one gets, up to a multiplicative numerical constant $c_{n, m}$, the following string of inequalities

$$
\begin{aligned}
\frac{\partial \sigma_{m}\left(u_{j \bar{k}}\right)}{\partial u_{i \bar{j}}} T_{\epsilon, i \bar{j}} & =c_{n, m} 1 / \epsilon^{2} d d^{c}\left(u_{(\epsilon)}-u\right) \wedge\left(d d^{c} u\right)^{m-1} \wedge \omega^{n-m} \\
& \geq c_{n, m} 1 / \epsilon^{2} \psi^{(m-1) / m}\left(\left(\psi^{1 / m}\right)_{(\epsilon)}-\psi^{1 / m}\right)=c_{n, m} \psi^{(m-1) / m} T_{\epsilon}\left(\psi^{1 / m}\right) .
\end{aligned}
$$

But $\psi$ is a strictly positive $\mathscr{C}^{1,1}$ function hence $T_{\epsilon}\left(\psi^{1 / m}\right) \geq-C_{1}\left(\|\psi\|,\left\|\psi^{1 / m}\right\|_{C^{1,1}}\right)$. Combining all those inequalities we obtain the claimed estimate.

From now on we drop the index $\epsilon$ in what follows. We will use the same calculations as in [Błocki and Dinew 2011], which in turn relied on [Trudinger 1980]. For some $\alpha, \beta \geq 2$ to be determined later set

$$
w:=\eta(T)^{\alpha}, \quad \text { where } \eta(z):=\left(1-|z|^{2}\right)^{\beta} .
$$

Then

$$
w_{i}=\eta_{i}(T)^{\alpha}+\alpha \eta(T)^{\alpha-1}(T)_{i}
$$

and

$$
\begin{aligned}
\frac{\partial \sigma_{m}\left(u_{j \bar{k}}\right)}{\partial u_{i \bar{j}}} w_{i \bar{j}}=\alpha \eta(T)^{\alpha-1} \frac{\partial \sigma_{m}\left(u_{j \bar{k}}\right)}{\partial u_{i \bar{j}}}(T)_{i \bar{j}}+\alpha(\alpha-1) \eta(T)^{\alpha-2} \frac{\partial \sigma_{m}\left(u_{j \bar{k}}\right)}{\partial u_{i \bar{j}}}(T)_{i}(T)_{\bar{j}} & +2 \alpha(T)^{\alpha-1} \operatorname{Re}\left(\frac{\partial \sigma_{m}\left(u_{j \bar{k}}\right)}{\partial u_{i \bar{j}}} \eta_{i}(T)_{\bar{j}}\right)+(T)^{\alpha} \frac{\partial \sigma_{m}\left(u_{j \bar{k}}\right)}{\partial u_{i \bar{j}}} \eta_{i \bar{j}}
\end{aligned}
$$


By Lemma 4.3 and the Schwarz inequality for $t>0$,

$$
\begin{aligned}
& \frac{\partial \sigma_{m}\left(u_{j \bar{k}}\right)}{\partial u_{i \bar{j}}} w_{i \bar{j}} \geq-C_{1} \alpha \eta(T)^{\alpha-1}+\alpha(\alpha-1) \eta(T)^{\alpha-2} \frac{\partial \sigma_{m}\left(u_{j \bar{k}}\right)}{\partial u_{i \bar{j}}}(T)_{i}(T)_{\bar{j}} \\
& -t \alpha(T)^{\alpha-1} \frac{\partial \sigma_{m}\left(u_{j \bar{k}}\right)}{\partial u_{i \bar{j}}}(T)_{i}(T)_{\bar{j}}-\frac{1}{t} \alpha(T)^{\alpha-1} \frac{\partial \sigma_{m}\left(u_{j \bar{k}}\right)}{\partial u_{i \bar{j}}} \eta_{i} \eta_{\bar{j}}+(T)^{\alpha} \frac{\partial \sigma_{m}\left(u_{j \bar{k}}\right)}{\partial u_{i \bar{j}}} \eta_{i \bar{j}}
\end{aligned}
$$

Therefore with $t=(\alpha-1) \eta / T$ we get

$$
\frac{\partial \sigma_{m}\left(u_{j \bar{k}}\right)}{\partial u_{i \bar{j}}} w_{i \bar{j}} \geq-C_{1} \alpha \eta(T)^{\alpha-1}+(T)^{\alpha} \frac{\partial \sigma_{m}\left(u_{j \bar{k}}\right)}{\partial u_{i \bar{j}}}\left(\eta_{i \bar{j}}-\frac{\alpha}{\alpha-1} \frac{\eta_{i} \eta_{\bar{j}}}{\eta}\right) .
$$

We now have

$$
\begin{aligned}
\eta_{i} & =-\beta z_{i} \eta^{1-1 / \beta} \\
\eta_{i \bar{j}} & =-\beta \delta_{i \bar{j}} \eta^{1-1 / \beta}+\beta(\beta-1) \bar{z}_{i} z_{j} \eta^{1-2 / \beta}
\end{aligned}
$$

and thus

$$
\left|\eta_{i} \bar{j}\right|,\left|\frac{\eta_{i} \eta_{\bar{j}}}{\eta}\right| \leq C(\beta) \eta^{1-2 / \beta}
$$

Coupling the above inequalities we get

$$
\frac{\partial \sigma_{m}\left(u_{j \bar{k}}\right)}{\partial u_{i \bar{j}}} w_{i \bar{j}} \geq-C_{2}(T)^{\alpha-1}-C_{3} w^{1-2 / \beta}(T)^{2 \alpha / \beta} \sum_{i, j}\left|\frac{\partial \sigma_{m}\left(u_{j \bar{k}}\right)}{\partial u_{i \bar{j}}}\right| .
$$

Fix $q$ with $n / m<q<p / m(m-1$ ) (by our assumption on $p$ such a choice is possible). By Lemma 4.2 $\|T\|_{p}$ and $\|\Delta u\|_{p}$ are under control. By Calderón-Zygmund inequalities we control $\left\|u_{i}\right\|_{p}$ too. Observe that $\partial \sigma_{m}\left(u_{j \bar{k}}\right) / \partial u_{i \bar{j}}$ is a sum of products of $m-1$ factors of the type $u_{i \bar{j}}$, and therefore its $p /(m-1)$-norm is also under control. It follows that for

$$
\alpha=1+\frac{p}{q m}, \quad \beta=2\left(\frac{q m+p}{p-q m(m-1)}\right),
$$

we have

$$
\left\|\left(\frac{\partial \sigma_{m}\left(u_{j \bar{k}}\right)}{\partial u_{i \bar{j}}} w_{i \bar{j}}\right)_{-}\right\|_{q m} \leq C_{3}\left(1+\left(\sup _{B} w\right)^{1-2 / \beta}\right)
$$

where $f_{-}:=-\min (f, 0)$. By Theorem 2.10 we can find continuous $m$-subharmonic $v$ vanishing on $\partial B$ and such that

$$
\sigma_{m}\left(v_{i \bar{j}}\right)=\left(\left(u^{i \bar{j}} w_{i \bar{j}}\right)_{-}\right)^{m} .
$$


Then the weak Gårding inequality yields

$$
\begin{aligned}
\frac{\partial \sigma_{m}\left(u_{j \bar{k}}\right)}{\partial u_{i \bar{j}}} v_{i \bar{j}} & =c_{n, m}\left(d d^{c} u\right)^{m-1} \wedge d d^{c} v \wedge \omega^{n-m} \\
& \geq c_{n, m}\left(\sigma_{m}\left(u_{i \bar{j}}\right)^{(m-1) / m}\left(\sigma_{m}\left(v_{i \bar{j}}\right)\right)^{1 / m}\right) \geq \frac{1}{C_{4}}\left(\frac{\partial \sigma_{m}\left(u_{j \bar{k}}\right)}{\partial u_{i \bar{j}}} w_{i \bar{j}}\right)_{-} \\
& \geq-\frac{1}{C_{4}} \frac{\partial \sigma_{m}\left(u_{j \bar{k}}\right)}{\partial u_{i \bar{j}}} w_{i \bar{j}} .
\end{aligned}
$$

By maximum principle we obtain that $w \leq-C_{4} v$, since this inequality holds on $\partial B$. Applying the stability theorem (Theorem 2.8), with $u=0$, we get

$$
\begin{aligned}
\sup _{B} w \leq C_{4}\|v\| & \leq C_{5}\left(\left\|\sigma_{m}\left(v_{i \bar{j}}\right)\right\|_{q}^{1 / m}\right)=C_{5}\left\|\left(\frac{\partial \sigma_{m}\left(u_{z_{j} \bar{z}_{k}}\right)}{\partial u_{i \bar{j}}} w_{i \bar{j}}\right)_{-}\right\|_{q n} \\
& \leq C_{6}\left(1+\left(\sup _{B} w\right)^{1-2 / \beta}\right) .
\end{aligned}
$$

Therefore $w \leq C_{7}$ and thus

$$
T^{\alpha} \leq C_{7} / \eta
$$

which is the desired bound.

Remark 4.4. An analogous reasoning can be applied to the real $m$-Hessian equation (using Wang stability theorem and existence of weak solutions). It turns out that for $m<n / 2$ the corresponding exponent in the $W^{2, p}$ Sobolev space is equal to $n(m-1) / 2$. Observe that this improves the $m(n-1) / 2$ exponent obtained by different methods by Urbas [2001]. Whether this exponent is optimal is however still unclear and would require construction of suitable Pogorelov type Hessian examples.

\section{Acknowledgements}

The authors were partially supported by NCN grant 2011/01/B/ST1/00879. The first author was also supported by Polish ministerial grant "Iuventus Plus" and Kuratowski fellowship granted by the Polish Mathematical Society (PTM) and Polish Academy of Science (PAN).

\section{References}

[Bedford and Taylor 1976] E. Bedford and B. A. Taylor, "The Dirichlet problem for a complex Monge-Ampère equation", Invent. Math. 37:1 (1976), 1-44. MR $56 \# 3351$ Zbl 0315.31007

[Bedford and Taylor 1982] E. Bedford and B. A. Taylor, "A new capacity for plurisubharmonic functions”, Acta Math. 149:1-2 (1982), 1-40. MR 84d:32024 Zbl 0547.32012

[Błocki 2003] Z. Błocki, "Interior regularity of the degenerate Monge-Ampère equation", Bull. Austral. Math. Soc. 68:1 (2003), 81-92. MR 2004g:35075 Zbl 1067.35028

[Błocki 2005] Z. Błocki, "Weak solutions to the complex Hessian equation", Ann. Inst. Fourier (Grenoble) 55:5 (2005), 1735-1756. MR 2006e:32042 Zbl 1081.32023

[Błocki 2009] Z. Błocki, "Defining nonlinear elliptic operators for non-smooth functions", pp. 111-124 in Complex analysis and digital geometry (Uppsala, 2006), edited by M. Passare, Acta Univ. Upsaliensis Skr. Uppsala Univ. C Organ. Hist. 86, Uppsala Universitet, Uppsala, 2009. MR 2011i:32038 Zbl 1215.35081 
[Błocki and Dinew 2011] Z. Błocki and S. Dinew, "A local regularity of the complex Monge-Ampère equation", Math. Ann. 351:2 (2011), 411-416. MR 2012h:32046 Zbl 1237.32006

[Caffarelli et al. 1985] L. Caffarelli, L. Nirenberg, and J. Spruck, "The Dirichlet problem for nonlinear second-order elliptic equations, III: Functions of the eigenvalues of the Hessian”, Acta Math. 155:3-4 (1985), 261-301. MR 87f:35098 Zbl 0654.35031

[Chou and Wang 2001] K.-S. Chou and X.-J. Wang, "A variational theory of the Hessian equation”, Comm. Pure Appl. Math. 54:9 (2001), 1029-1064. MR 2002e:35072 Zbl 1035.35037

[Dinew 2009] S. Dinew, "An inequality for mixed Monge-Ampère measures”, Math. Z. 262:1 (2009), 1-15. MR 2009m:32065 Zbl 1169.32007

[Dinew and Kołodziej 2012] S. Dinew and S. Kołodziej, "Liouville and Calabi-Yau type theorems for complex Hessian equations", preprint, 2012. arXiv 1203.3995

[Gårding 1959] L. Gårding, "An inequality for hyperbolic polynomials”, J. Math. Mech. 8:6 (1959), 957-965. MR 22 \#4809 Zbl 0090.01603

[Hou 2009] Z. Hou, “Complex Hessian equation on Kähler manifold”, Int. Math. Res. Not. 2009:16 (2009), 3098-3111. MR 2010m:32026 Zbl 1177.32013

[Hou et al. 2010] Z. Hou, X.-N. Ma, and D. Wu, "A second order estimate for complex Hessian equations on a compact Kähler manifold", Math. Res. Lett. 17:3 (2010), 547-561. MR 2011j:32030 Zbl 1225.32026

[Ivochkina et al. 2004] N. Ivochkina, N. Trudinger, and X.-J. Wang, "The Dirichlet problem for degenerate Hessian equations", Comm. Partial Differential Equations 29:1-2 (2004), 219-235. MR 2005d:35076 Zbl 1140.35418

[Jbilou 2010] A. Jbilou, "Équations Hessiennes complexes sur des variétés Kählériennes compactes", C. R. Math. Acad. Sci. Paris 348:1-2 (2010), 41-46. MR 2011a:32040 Zbl 1189.53071

[Kokarev 2010] V. N. Kokarev, “Смешанные формы обьема и комплексное уравнение типа Монжа-Ампера на Кэлеровых многообразиях положительной кривизны”, Izv. Ross. Akad. Nauk Ser. Mat. 74:3 (2010), 65-78. Translated as "Mixed volume forms and a complex equation of Monge-Ampère type on Kähler manifolds of positive curvature" in Izv. Math. 74:3 (2010), 501-514. MR 2011k:32055 Zbl 1200.32015

[Kołodziej 1996] S. Kołodziej, "Some sufficient conditions for solvability of the Dirichlet problem for the complex MongeAmpère operator", Ann. Polon. Math. 65:1 (1996), 11-21. MR 98a:32015 Zbl 0878.32014

[Kołodziej 1998] S. Kołodziej, “The complex Monge-Ampère equation”, Acta Math. 180:1 (1998), 69-117. MR 99h:32017 Zbl 0913.35043

[Kołodziej 2002] S. Kołodziej, "Equicontinuity of families of plurisubharmonic functions with bounds on their Monge-Ampère masses", Math. Z. 240:4 (2002), 835-847. MR 2003f:32043 Zbl 1014.32023

[Kołodziej 2003] S. Kołodziej, “The Monge-Ampère equation on compact Kähler manifolds”, Indiana Univ. Math. J. 52:3 (2003), 667-686. MR 2004i:32062 Zbl 1039.32050

[Kołodziej 2005] S. Kołodziej, "The complex Monge-Ampère equation and pluripotential theory", Mem. Amer. Math. Soc. 178:840 (2005). MR 2006d:32054 Zbl 1084.32027

[Krylov 1995] N. V. Krylov, "On the general notion of fully nonlinear second-order elliptic equations", Trans. Amer. Math. Soc. 347:3 (1995), 857-895. MR 95f:35075 Zbl 0832.35042

[Labutin 2002] D. A. Labutin, "Potential estimates for a class of fully nonlinear elliptic equations", Duke Math. J. 111:1 (2002), 1-49. MR 2002m:35053 Zbl 1100.35036

[Li 2004] S.-Y. Li, "On the Dirichlet problems for symmetric function equations of the eigenvalues of the complex Hessian", Asian J. Math. 8:1 (2004), 87-106. MR 2006d:32057 Zbl 1068.32024

[Trudinger 1980] N. S. Trudinger, "Local estimates for subsolutions and supersolutions of general second order elliptic quasilinear equations", Invent. Math. 61:1 (1980), 67-79. MR 81m:35058 Zbl 0453.35028

[Trudinger 1995] N. S. Trudinger, "On the Dirichlet problem for Hessian equations", Acta Math. 175:2 (1995), 151-164. MR 96m:35113 Zbl 0887.35061

[Trudinger and Wang 1999] N. S. Trudinger and X.-J. Wang, "Hessian measures, II", Ann. of Math. (2) 150:2 (1999), $579-604$. MR 2001f:35141 Zbl 0947.35055 
[Urbas 2001] J. Urbas, "An interior second derivative bound for solutions of Hessian equations", Calc. Var. Partial Differential Equations 12:4 (2001), 417-431. MR 2002g:35070 Zbl 0986.35035

[Wang 2009] X.-J. Wang, "The $k$-Hessian equation", pp. 177-252 in Geometric analysis and PDEs (Cetraro, 2007), edited by M. J. Gursky et al., Lecture Notes in Math. 1977, Springer, Dordrecht, 2009. MR 2010f:35105 Zbl 1196.35093

Received 6 Feb 2013. Accepted 27 Nov 2013.

SŁAWOMIR Dinew: Slawomir.Dinew@im.uj.edu.pl

Mathematics Department, Rutgers Unversity, Newark, NJ 07102, United States

and

Department of Mathematics and Computer Science, Jagiellonian University, ul. Lojasiewicza 6, 30-348 Krakow, Poland

SŁaWOMIR KoŁOdZIEJ: slawomir.kolodziej@im.uj.edu.pl

Department of Mathematics and Computer Science, Jagiellonian University, ul Lojasiewicza 6, 30-348 Krakow, Poland 


\title{
Analysis \& PDE
}

\author{
msp.org/apde
}

\section{EDITORS}

EDITOR-IN-CHIEF

\author{
Maciej Zworski \\ zworski@math.berkeley.edu \\ University of California \\ Berkeley, USA
}

BOARD OF EDITORS

Nicolas Burq Université Paris-Sud 11, France

nicolas.burq@math.u-psud.fr

Sun-Yung Alice Chang Princeton University, USA

chang@math.princeton.edu

Michael Christ University of California, Berkeley, USA

mchrist@math.berkeley.edu

Charles Fefferman Princeton University, USA

cf@math.princeton.edu

Ursula Hamenstaedt Universität Bonn, Germany

ursula@math.uni-bonn.de

Vaughan Jones U.C. Berkeley \& Vanderbilt University vaughan.f.jones@vanderbilt.edu

Herbert Koch Universität Bonn, Germany koch@math.uni-bonn.de

Izabella Laba University of British Columbia, Canada ilaba@math.ubc.ca

Gilles Lebeau Université de Nice Sophia Antipolis, France lebeau@unice.fr

László Lempert Purdue University, USA lempert@math.purdue.edu

Richard B. Melrose Massachussets Institute of Technology, USA rbm@math.mit.edu

Frank Merle Université de Cergy-Pontoise, France Frank.Merle@u-cergy.fr

William Minicozzi II Johns Hopkins University, USA minicozz@math.jhu.edu

Werner Müller Universität Bonn, Germany mueller@math.uni-bonn.de
Yuval Peres

Gilles Pisier

Tristan Rivière

Igor Rodnianski

Wilhelm Schlag

Sylvia Serfaty

Yum-Tong Siu

Terence Tao

Michael E. Taylor

Gunther Uhlmann

András Vasy

Dan Virgil Voiculescu

Steven Zelditch
University of California, Berkeley, USA

peres@stat.berkeley.edu

Texas A\&M University, and Paris 6

pisier@math.tamu.edu

ETH, Switzerland

riviere@math.ethz.ch

Princeton University, USA

irod@math.princeton.edu

University of Chicago, USA

schlag@math.uchicago.edu

New York University, USA

serfaty@cims.nyu.edu

Harvard University, USA

siu@math.harvard.edu

University of California, Los Angeles, USA

tao@math.ucla.edu

Univ. of North Carolina, Chapel Hill, USA

met@math.unc.edu

University of Washington, USA

gunther@math.washington.edu

Stanford University, USA

andras@math.stanford.edu

University of California, Berkeley, USA

dvv@math.berkeley.edu

Northwestern University, USA

zelditch@math.northwestern.edu

\section{PRODUCTION}

production@msp.org

Silvio Levy, Scientific Editor

See inside back cover or msp.org/apde for submission instructions.

The subscription price for 2014 is US $\$ 180 /$ year for the electronic version, and $\$ 355 /$ year $(+\$ 50$, if shipping outside the US) for print and electronic. Subscriptions, requests for back issues from the last three years and changes of subscribers address should be sent to MSP.

Analysis \& PDE (ISSN 1948-206X electronic, 2157-5045 printed) at Mathematical Sciences Publishers, 798 Evans Hall \#3840, c/o University of California, Berkeley, CA 94720-3840, is published continuously online. Periodical rate postage paid at Berkeley, CA 94704, and additional mailing offices.

APDE peer review and production are managed by EditFLOW ${ }^{\circledR}$ from Mathematical Sciences Publishers.

PUBLISHED BY

- mathematical sciences publishers

nonprofit scientific publishing

http://msp.org/

(C) 2014 Mathematical Sciences Publishers 


\section{ANALYSIS \& PDE}

\section{Volume $7 \quad$ No. $1 \quad 2014$}

PAUl LAURAIN and TRISTAN RIVIÈRE

Global well-posedness of slightly supercritical active scalar equations

Michael Dabkowski, AleXander Kiselev, Luis Silvestre and Vlad Vicol

The nonlinear Schrödinger equation ground states on product spaces

Susanna TerRacini, Nikolay TzVetkov and Nicola Visciglia

Orthonormal systems in linear spans

ALLISON LEWKO and MARK LEWKO

A partial data result for the magnetic Schrödinger inverse problem

FRANCIS J. CHUNG

Sharp polynomial decay rates for the damped wave equation on the torus

NALini ANANTHARAMAN and MATTHIEU LÉAUTAUd

The $J$-flow on Kähler surfaces: a boundary case

Hao Fang, Mijia Lai, Jian Song and Ben Weinkove

A priori estimates for complex Hessian equations

SŁAWOMIR Dinew and SŁAWOMIR KoŁOdZIEJ

The Aharonov-Bohm effect in spectral asymptotics of the magnetic Schrödinger operator

GREGORY ESKIN and JAMES RALSTON 